\title{
Investigation on the Effect of NiZn Ferrite on the Mechanical and Thermal Conductivity of PLA/LNR Nanocomposites
}

\author{
Mou'ad A. Tarawneh, Dalila Shahdan, and Sahrim Hj Ahmad \\ School of Applied Physics, Faculty of Science and Technology, National University of Malaysia, 43600 Bangi, Selangor, Malaysia \\ Correspondence should be addressed to Mou’ad A. Tarawneh; moaath20042002@yahoo.com
}

Received 10 April 2013; Revised 5 October 2013; Accepted 6 October 2013

Academic Editor: Sheng-Rui Jian

Copyright (c) 2013 Mou'ad A. Tarawneh et al. This is an open access article distributed under the Creative Commons Attribution License, which permits unrestricted use, distribution, and reproduction in any medium, provided the original work is properly cited.

\begin{abstract}
The mechanical and conductivity of magnetic polymer nanocomposite (MPNC) of nickel zinc (NiZn) ferrite nanoparticles incorporated with poly(lactic acid) (PLA) and liquid natural rubber (LNR) as compatibilizer is reported. The matrix was prepared from PLA and LNR in the ratio of $90: 10$. The MPNC of PLA/LNR/NiZn ferrite then was prepared via Thermo Haake internal mixer using melt-blending method from different filler loading from 1-5 wt $\%$ NiZn ferrite. The result of tensile tests showed that as the filler loading increases, the tensile strength also increases until an optimum value of filler loading was reached. Young's modulus, tensile strength, and elongation at break have also increased. The study proves that NiZn ferrite is an excellent reinforcement filler in PLA/LNR matrix. The optimum thermal conductivity of PLA/LNR composites achieved with ( 4 wt $\%$ NiZn) due to the effective combination of NiZn-NiZn conductive networks. The scanning electron micrograph (SEM) reveal that the aspect ratio and filler orientation in the PLA/LNR matrix also strongly promoted interfacial adhesion between the filler and the matrix to control its properties.
\end{abstract}

\section{Introduction}

Poly(lactic acid) (PLA) has received great attention due to its biodegradable, renewability, and comparable properties with petroleum-based polymers. Recent concerns on the environmental effect have fuelled the need to explore new alternatives to the commodity plastics. The use of nondegradable plastics for consumer goods such as packaging material has created so much disposal problem. Apart from the environmental issues, the cost of production has been escalating due to its dependent on the oil prices. As such, polymers from renewable sources are on the edge to compete with the traditional plastics.

PLA is a typical biodegradable polyester obtained by synthesis of lactic acid (or lactide), which can be produced from renewable resources such as corn or sugarcane [1]. It is a sustainable alternative to petrochemical-derived products. PLA uses have been limited to specialty biomedical niches, such as resorbable sutures [2] in a decade ago. Presently, a great deal of research work on plastic materials has been dedicated to the application of biodegradable materials. It is now gaining popularity as it has been accepted in many application areas such as food packaging, bags and sacks, loose-fill packaging agricultural film, and some specialty application [3].

Magnetic polymer nanocomposite (MPNC) has been one of the most interesting topics to be studied, nowadays. This is due to its unique properties which can play a role in various applications. Whilst, with regard to the awareness of environmental issues, a lot of study related to biodegradable or bio-based polymer had been rapidly done, and polymer matrix nanocomposite is one of it. This topic may cover a various range of application such as biomedical, electrical conductivity, barrier properties, membrane separation, UV screens, flammability resistance, polymer blend compatibilization, and many more.

Nowadays, to enhance some of the above-mentioned properties, polymers have been filled with fillers in nanosized. This is due to its very small size with high surface area, and it could improve the physical properties of polymers. Ferromagnetic materials (ferrites) are materials with various potential applications such as antennas, permanent 
magnets, memory storage devices, microwave devices, and telecommunication equipment [4]. This magnetic nanoparticles (MNPs) which incorporated with polymer matrix could produce a unique fundamental properties associated with them such as quantum tunnelling of magnetization, quantum size effects, and spin reversals in single domain particles (superparamagnetism phenomena) [5]. As stated by [6], nickel zinc (NiZn) ferrites are magnetically soft materials that possess versatile properties like high saturation magnetization, low coercivity, high resistivity, and low dielectric losses, and these properties make them best suited for numerous high frequency applications like inductance coils, transformer cores, antennas, and microwave absorbers. In this study, NiZn ferrite was used as filler due to its excellent magnetic properties, high electrical resistivity, mechanical hardness, chemical stability, thermal conductivity, and cost effectiveness [7]. In order to get a homogeneous and uniform distribution, an assistance of ultrasonic treatment had been used. Indirect mixing technique was done to prepare the LNR and NiZn ferrites which later on will be mixed together with PLA in internal mixer.

Polymer blend was prepared along with liquid natural rubber (LNR). The addition of low molecular weight reactive LNR can improve fracture toughness and impact strength of PLA [8]. Other than that, it will act as compatibilizer and toughening agent. The LNR with some active terminals like $-\mathrm{OH}$ is expected to react with the plastic particles and thereby bond the plastic particles to the natural rubber matrix [9]. Thus, the assistance of LNR is expected not just to improve the bonding between the polymer matrix and NR but also between the PLA and NiZn. Tensile properties of the blend are determined by the homogeneity of mixing; that is, the properties are better with higher homogeneity [10]. In addition, mechanical properties of polymer blends are also strongly dependent on the processing method and sample. As for that, for polymer blending which is usually an immiscible blend, the components are compatibilize to improve its physical properties.

In this research study, poly(lactic acid) (PLA)/liquid natural rubber (LNR)/NiZn ferrite nanocomposites were prepared by melt blending method. By applying this method, NiZn ferrite will be dispersed uniformly or homogeneously with the help of LNR in the polymer matrix to enhance the thermal properties of this nanocomposite. The thermal conductivity was measured by using laser flash diffusivity method. This parameter is critical for the basic understanding of the heat dissipation on the surface of NiZn ferrite in a PLA/LNR composite. Thus, PLA/LNR was used as a base to produce a PLA/LNR/NiZn nanocomposite at different NiZn ferrite content. The effect in terms of thermal behavior on the different content of NiZn ferrite in PLA/LNR environment is reported.

\section{Materials and Methods}

2.1. Materials. A commercial poly(lactic acid) of Natureworks Ingeo Biopolymer 3251D, purchased from Unic Technology Ltd. China, is used as the thermoplastic resin in this study. It has density of $1.24 \mathrm{~g} / \mathrm{cm}^{3}$ and melt flow index of $30-40 \mathrm{~g} / 10 \mathrm{~min}\left(190^{\circ} \mathrm{C} / 2,16 \mathrm{~kg}\right)$ and melting temperature in between $188-210^{\circ} \mathrm{C}$. SMR-L grade natural rubber (NR) was obtained from the Malaysian Rubber Board (MRB). The liquid natural rubber (LNR) was synthesized using photochemical sensitized degradation/oxidation technique on natural rubber in our polymer processing laboratory at School of Applied Physics, UKM. Nickel zinc (NiZn) ferrite nanoparticle powder of the chemical formula $\mathrm{Ni}_{0.5} \mathrm{Zn}_{0.5} \mathrm{Fe}_{2} \mathrm{O}_{4}$ with $98.5 \%$ purity and $10-30 \mathrm{~nm}$ average particles size was supplied by Nanostructured and Amorphous Materials Inc., USA.

2.2. Material Preparation. PLA blends were prepared using an internal mixer (Haake Rheomix 600P) at the temperature of $180^{\circ} \mathrm{C}$ with rotor speed of $100 \mathrm{rpm}$ for $15 \mathrm{~min}$ of mixing time. Indirect mixing technique (IDT) was applied in order to prepare nanocomposites which involved mixing the NiZn ferrite with LNR separately by ultrasonic treatment for 1 hour, before it was melt blended with PLA in the mixer. Before mixing, the PLA was dried in the oven at $100^{\circ} \mathrm{C}$ for 24 hours to eliminate moisture. The nanocomposites were prepared by melt blending process of PLA with LNR and NiZn ferrite in a ratio of $90 \mathrm{wt} \%$ PLA, $10 \mathrm{wt} \%$ LNR as compatibilzer and 1, 2, 3, 4, and 5 wt $\%$ NiZn ferrite. PLA was first discharged into the mixer and allowed to melt for about 3 min before the LNR and NiZn ferrite were added.

2.3. Characterizations. The tensile testing was done using Instron Tensile Machine with $5 \mathrm{kN}$ load cell according to ASTM 412 standard procedure using test specimens of $1 \mathrm{~mm}$ thickness and a crosshead speed $50 \mathrm{~mm} \mathrm{~min}^{-1}$. At least five samples were tested for each composition.

Differential scanning calorimeter (DSC) analysis was done on PLA/LNR nanocomposites samples to identify their glass transition temperature. The measurement was carried out using DSC 882 (Mettler Toledo). Heating measurement was done from $-60^{\circ} \mathrm{C}$ to $250^{\circ} \mathrm{C}$, ramp $10^{\circ} \mathrm{C} / \mathrm{min}$. The sample weight is about $20 \mathrm{mg}$.

The thermal conductivity was measured by the laser flash method. Disk-type samples $(12.7 \mathrm{~mm}$ in diameter and $1 \mathrm{~mm}$ in thickness) were set in an electric furnace. Specific heat capacities were measured with a differential scanning calorimeter DSC. Thermal conductivity $\left(\lambda, \mathrm{Wm}^{-1} \mathrm{~K}^{-1}\right)$ was calculated from thermal diffusivity $\left(\alpha, \mathrm{m}^{2} \mathrm{~s}^{-1}\right)$, density $(\rho$, $\mathrm{kg} \mathrm{cm}^{-3}$ ), and specific heat capacity $\left(C, \mathrm{Jg}^{-1} \mathrm{~K}^{-1}\right)$ at each temperature using the following:

$$
\lambda=\alpha \cdot \rho \cdot C
$$

The reference used for the heat capacity calculation was a $12.7 \mathrm{~mm}$ thick specimen of pyroceram. The thermal conductivity of PLA/LNR nanocomposites of all volume fractions was studied from $30^{\circ} \mathrm{C}$ to $180^{\circ} \mathrm{C}$.

Morphology of the PLA/LNR and PLA/LNR nanocomposites was examined by scanning electron microscope (Philips XL 30). The samples were coated with a thin layer of gold to avoid electrostatic charging during examination. 


\section{Results and Discussion}

The effect of filler loading of nickel zinc ferrite on tensile properties is shown in Table 1. The result indicated that the tensile strength increases with increasing amount of NiZn ferrites. The optimum tensile strength is obtained at $4 \mathrm{wt} \%$, with an increasing of $20 \%$ compared to the nonfiller PLA/LNR blends. The incorporation of NiZn ferrites as filler loading actually is associated with the improvement of tensile strength. The effect of good interface between NiZn ferrites and PLA is very important to the material to stand the stress. In this study, LNR which acts as compatibilizer contributes to the improvement of filler dispersion. The LNR with some active terminals like $-\mathrm{OH}$ is expected to react with the plastic particles and thereby bond the plastic particles to the natural rubber matrix [11]. When load is applied, the matrix will distribute the force to NiZn ferrites which carry most of the applied load. As for that, the lower value of tensile strength at lower filler loading is due to the flaws created by the filler loading. This flaw acts as stress concentration and causes the bond between the filler and matrix to break. While at higher volume fractions, the matrix is sufficiently restrained, and the stress is more evenly distributed. However, when the content of filler loading is higher than the optimum value, the NiZn ferrite could not able to be dispersed well in the matrix. As a result, it tends to agglomerate into big cluster due to the surface energy of NiZn ferrites in which become the main cause of decreasing value of tensile strength. Compared with the nonfiller PLA/LNR, Young's modulus increased when the amount of filler loading is increased, then the value is dropped due to the excessive amount of filler loading in polymer matrix as shown in Table 1 . The highest value is obtained at $4 \mathrm{wt} \%$ of NiZn ferrite, with $30 \%$ of percentage increase. The improvement of the modulus is due to the existence of NiZn ferrites nanoparticles with a good dispersion of the filler in PLA matrix. Young's modulus of higher filler loading decreased due to the increase of aggregation of NiZn ferrites in PLA/LNR matrix, and this can be revealed in SEM analysis later on. The addition of LNR was found to improve the mechanical properties probably due to the existence of rubber particles in the PLA [11]. In Table 1, it shows the elongation at break of PLA/LNR and PLA/LNR with different amount of filler loading. The percentage of elongation at break of nanocomposites can be seen with the increasing of NiZn ferrites. As the amount of filler loading increases, the tensile stress level gradually increases, but at the same time the tensile strain of nanocomposites decreased. The filler will act as physical or chemical cross-linking points and restricts the movement of polymer chains. It is a sign of the material flexibility, which shows that the addition of NiZn ferrites creates a stronger but yet brittle nanocomposites [12].

The optimization of PLA/LNR-filler interfacial interaction is significant for the enhancement of thermal transport of PLA/LNR composite. Figure 1 reveals the thermal conductivity measured at various temperature points in the range of $30^{\circ} \mathrm{C}-180^{\circ} \mathrm{C}$ of PLA/LNR nanocomposites filled with different percent of NiZn ferrites. It can be observed that the thermal conductivity of PLA/LNR with $4 \mathrm{wt} \% \mathrm{NiZn}$ is larger than pure PLA/LNR or PLA/LNR with another percent. Thermal

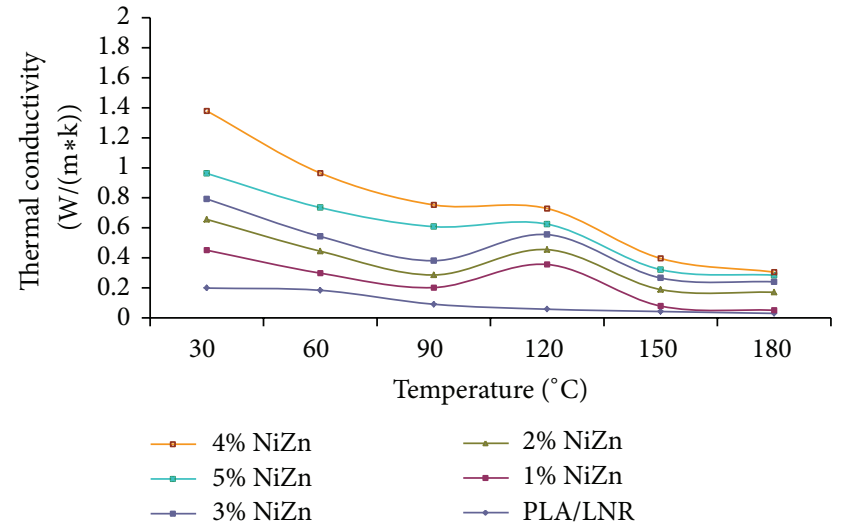

FIGURE 1: The thermal conductivity of PLA/LNR and PLA/LNR nanocomposites.

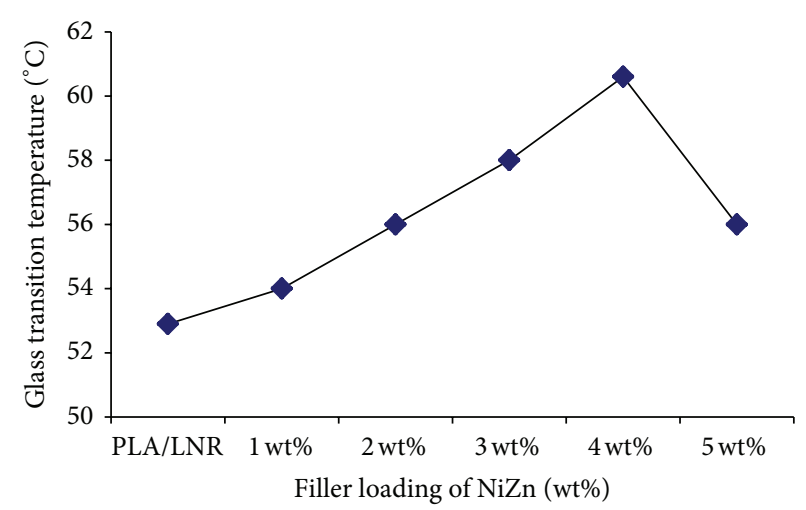

Figure 2: The glass transition temperature $\left({ }^{\circ} \mathrm{C}\right)$ of PLA/LNR and PLA/LNR nanocomposites.

TABLE 1: Tensile properties of PLA/LNR with different wt $\%$ of filler loading.

\begin{tabular}{lccc}
\hline Sample & $\begin{array}{c}\text { Young's modulus } \\
(\mathrm{MPa})\end{array}$ & $\begin{array}{c}\text { Tensile strength } \\
(\mathrm{MPa})\end{array}$ & $\begin{array}{c}\text { Elongation at } \\
\text { break (\%) }\end{array}$ \\
\hline PLA/LNR & 5047.41 & 35.97 & 11.8 \\
1 wt\% NiZn & 6181.34 & 36.85 & 4.83 \\
2 wt\% NiZn & 6227.25 & 37.71 & 4.82 \\
3 wt\% NiZn & 6253.45 & 39.62 & 2.54 \\
4 wt\% NiZn & 6553.41 & 42.79 & 2.74 \\
5 wt\% NiZn & 5926.14 & 37.71 & 1.63 \\
\hline
\end{tabular}

transport in the NiZn nanocomposites includes phonon diffusion in the matrix and ballistic transportation in the filler. The improving of thermal conductivity in PLA/LNR/4 wt\% NiZn may stem from the improved percolation because of better dispersion and formation of a network [13]. A key issue in producing superior PLA/LNR/NiZn nanocomposites for thermally conductive applications appears to be the ability to control aggregation of NiZn in PLA/LNR matrix to obtain an interconnecting network suitable for heat transfer [14]. Therefore, the large amount of junctions among the NiZn 


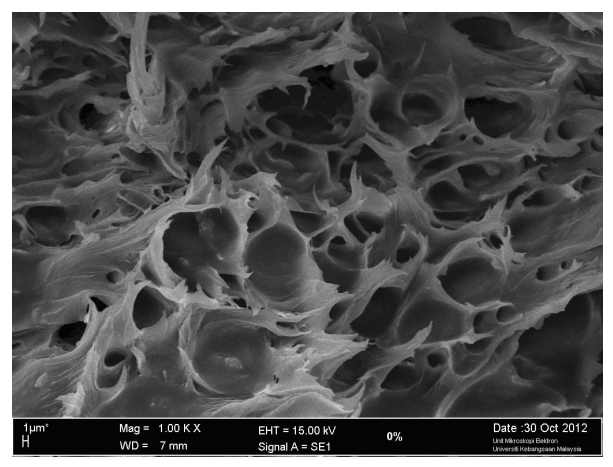

(a)

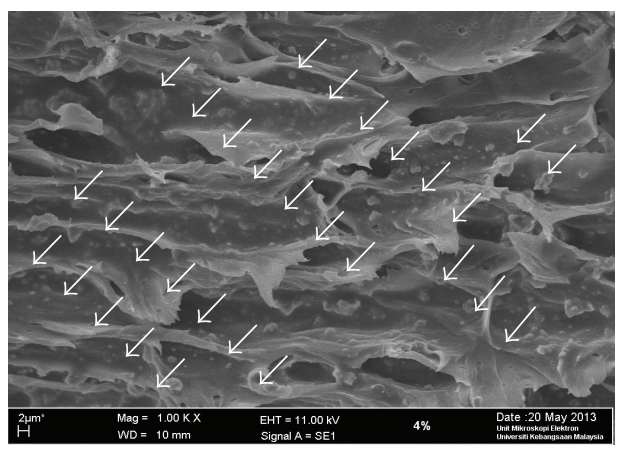

(c)

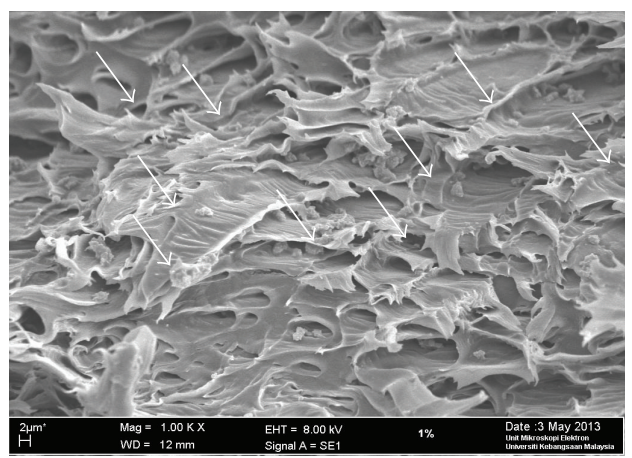

(b)

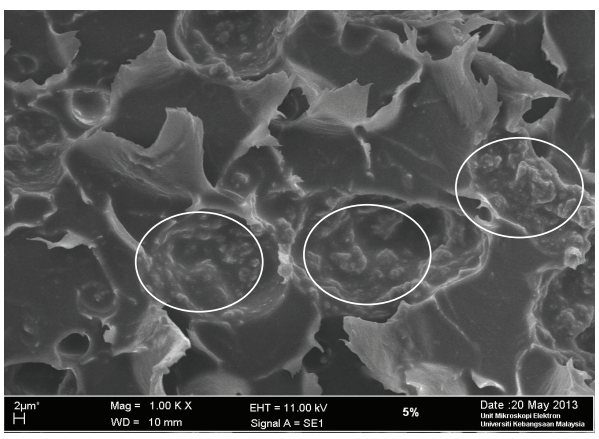

(d)

Figure 3: SEM micrograph of (a) PLA/LNR, (b) 1\% NiZn ferrites, (c) $4 \%$ NiZn ferrites, and (d) 5\% NiZn ferrites.

which forms a conducting path in the matrix is believed to be the reason causing the increase in thermal conductivity by obtaining close molecular contact and corresponding to a relatively high efficiency in transferring thermal energy from the PLA/LNR to the PLA/LNR.

The effect reducing the thermal conductivity is the transfer of phonons from NiZn to NiZn. This transition occurs by direct coupling between the nanofiller, in case of the agglomeration of NiZn, or via the matrix. In all these cases, the transition occurs via an interface, and thus the coupling losses can be assorted to an intense phonon boundary scattering. At the same time, the thermal conductivity decreased with increasing the temperature (if the temperature is near from the melting point of the matrix). This indicates that the thermal conductivity of the composites is mainly dominated by the interface thermal transport between NiZn/matrix or NiZn/NiZn interface. Thus, it is believed that the decreased effective thermal conductivity of the studied composites could be due to high interface thermal resistance across the $\mathrm{NiZn/matrix} \mathrm{or} \mathrm{NiZn/NiZn} \mathrm{interfaces} \mathrm{[15].}$

In addition, as we see from Figure 2, it was also found that the glass transition temperature $\left(T_{g}\right)$ of the PLA/LNR nanocomposites is higher at $4 \mathrm{wt} \% \mathrm{NiZn}$, which is due to the nanofiller $(\mathrm{NiZn})$ being more uniformly dispersed in the matrix. Therefore, the interfacial area is larger, and the interaction between the matrix and nanofiller is stronger. The rise in $T_{g}$ in any polymeric system is associated with a restriction in molecular motion, reduction in free volume and/or higher degree of crosslinking due to the interactions between the polymer chains and the nanoparticles, and the reduction of macromolecular chain mobility [16]. When the dispersion of the filler is higher in the matrix, it will create a network of nanoparticles which make the macromolecules move hardly, and the $T_{g}$ of the matrix is increased. In other words, the presence of NiZn has such a dramatic effect on the relaxation processes, typical for the glassy state of PLA/LNR, that the dominating deformation mechanisms become totally different. These relaxation processes are also often associated with the ductile-brittle transition, which manifest themselves in a rather sharp reduction of local mobility of polymer chains [17]. However, after $4 \mathrm{wt} \%$, the conglomerations and matrix holes existing in the network of NiZn may perform as defects, which enable the macromolecules to move easily; therefore, the $T_{g}$ of the matrix is lower.

Scanning electron micrograph (SEM) illustrated in Figure 3, shows the homogeneity dispersion of nanoparticles within the matrix PLA/LNR composites. The presence of NiZn ferrite can be distinguished from the micrograph due to their bright phase and spherical shape. Figure 3(a) shows $\mathrm{PLA} / \mathrm{LNR}$ with $0 \mathrm{wt} \%$ of NiZn ferrites, and this figure shows a heterogeneous morphology surfaces resulted in the surfaces. The holes observed in the micrograph indicate that the rubber particles dispersed in the PLA matrix. The cavitations show that the addition of liquid rubber acts as the toughening agent to the PLA matrix. However, in Figure 3(b) the presence of NiZn ferrites can be seen with good particles dispersion within the matrix. The good dispersion is due to the nanoparticles-polymer interactions, which have improved 
the expense of the interparticle interactions due to agglomeration and hence formed a uniform interconnected network within the host. The assistance of ultrasonic treatment during the indirect mixing technique of LNR and NiZn ferrites also contributes to the well dispersion of filler into the matrix. This supports that the earlier explanation of NiZn ferrite assisted in enhancing the properties of PLA/LNR. At $4 \mathrm{wt} \%$ of NiZn ferrites (Figure 3(c)), it is observed that the filler dispersed uniformly. This support earlier explanation of optimum strength value obtained is at $4 \mathrm{wt} \% \mathrm{NiZn}$ ferrites. However, poor dispersion of $5 \mathrm{wt} \%$ nanoparticles can be seen in Figure 3(d), which clearly shows poor polymernanoparticles adhesion that attributed to the reduction of the nanocomposites properties. The filler shows formation of bigger agglomeration.

\section{Conclusion}

NiZn ferrite nanoparticles reinforced PLA/LNR composites prepared via melt blending method. Mechanical tests show that compared with PLA/LNR, the tensile strength and Young's modulus are improved significantly by incorporating $\mathrm{NiZn}$. The laser flash technique was used to measure the thermal conductivity from the results obtained; the high thermal conductivity was achieved at $4 \mathrm{wt} \%$ of NiZn compared with PLA/LNR due to the effective combination of nanoparticles conductive networks. The LNR that acts as compatibilizer helps in improving the properties and at the same time improved the dispersion of filler loading onto the matrix. Homogeneous dispersion of NiZn throughout the matrix and strong interfacial adhesion between NiZn and matrix as confirmed by SEM images are proposed to be responsible for significant mechanical and thermal enhancement.

\section{Acknowledgments}

The authors gratefully thank the financial support provided by faulty of science and technology (FST), UKM, under grant no. UKM-DLP-2012-029. The authors also appreciate the cooperation given by the staff of Electron Microscopy Unit, UKM, during the SEM-utilization experiments.

\section{References}

[1] E. T. H. K. R. Vink, D. A. Rábago, P. R. Glassner, and P. R. Gruber, "Applications of life cycle assessment to NatureWorks polylactide (PLA) production," Polymer Degradation and Stability, vol. 80, pp. 403-419, 2003.

[2] D. W. Grijpma, H. Altpeter, M. J. Bevis, and J. Feijen, "Improvement of the mechanical properties of poly(D,L-lactide) by orientation," Polymer International, vol. 51, no. 10, pp. 845-851, 2002.

[3] D. Platt, Biodegradable Polymers, Smithers Rapra, 2006.

[4] X. Chen, S. Wei, A. Yadav et al., "Poly(propylene)/carbon nanofiber nanocomposites: ex situ solvent-assisted preparation and analysis of electrical and electronic properties," Macromolecular Materials and Engineering, vol. 296, no. 5, pp. 434443, 2011.
[5] M. N. Akhtar, N. Yahya, K. Koziol, and N. Nasir, "Synthesis and characterizations of $\mathrm{Ni}_{0.8} \mathrm{Zn}_{0.2} \mathrm{Fe}_{2} \mathrm{O}_{4}$-MWCNTs composites for their application in sea bed logging," Ceramics International, vol. 37, no. 8, pp. 3237-3245, 2011.

[6] M. H. Flaifel, S. H. Ahmad, M. H. Abdullah, and B. A. Al-Asbahi, "NiZn ferrite filled thermoplastic natural rubber nanocomposites: effect of low temperature on their magnetic behavior," Cryogenics, vol. 52, pp. 523-529, 2012.

[7] P. P. Sarangi, S. R. Vadera, M. K. Patra, and N. N. Ghosh, "Synthesis and characterization of pure single phase Ni-Zn ferrite nanopowders by oxalate based precursor method," Powder Technology, vol. 203, no. 2, pp. 348-353, 2010.

[8] S. H. Ahmad, R. Rasid, N. N. Bonnia et al., "Polyester-kenaf composites: effects of alkali fiber treatment and toughening of matrix using liquid natural rubber," Journal of Composite Materials, vol. 45, no. 2, pp. 203-217, 2011.

[9] A. Ibrahim and M. Dahlan, "Thermoplastic natural rubber blends," Progress in Polymer Science, vol. 23, no. 4, pp. 665-706, 1998.

[10] H. M. Dahlan, M. D. Khairul Zaman, and A. Ibrahim, "The morphology and thermal properties of liquid natural rubber (LNR)-compatibilized 60/40 NR/LLDPE blends," Polymer Testing, vol. 21, no. 8, pp. 905-911, 2002.

[11] M. Jacob, S. Thomas, and K. T. Varughese, "Mechanical properties of sisal/oil palm hybrid fiber reinforced natural rubber composites," Composites Science and Technology, vol. 64, no. 7-8, pp. 955-965, 2004.

[12] S. H. Jin, Y.-B. Park, and K. H. Yoon, "Rheological and mechanical properties of surface modified multi-walled carbon nanotube-filled PET composite," Composites Science and Technology, vol. 67, no. 15-16, pp. 3434-3441, 2007.

[13] S. Kumar, M. A. Alam, and J. Y. Murthy, "Effect of percolation on thermal transport in nanotube composites," Applied Physics Letters, vol. 90, no. 10, Article ID 104105, 3 pages, 2007.

[14] R. Prasher, "Thermal boundary resistance and thermal conductivity of multiwalled carbon nanotubes," Physical Review B, vol. 77, no. 7, Article ID 075424, 11 pages, 2008.

[15] R. Sivakumar, S. Guo, T. Nishimura, and Y. Kagawa, "Thermal conductivity in multi-wall carbon nanotube/silica-based nanocomposites," Scripta Materialia, vol. 56, no. 4, pp. 265-268, 2007.

[16] P. Pötschke, T. D. Fornes, and D. R. Paul, "Rheological behavior of multiwalled carbon nanotube/polycarbonate composites," Polymer, vol. 43, no. 11, pp. 3247-3255, 2002.

[17] A. E. Bogdanovich and P. D. Bradford, "Carbon nanotube yarn and 3-D braid composites-part I: tensile testing and mechanical properties analysis," Composites A, vol. 41, no. 2, pp. 230-237, 2010. 

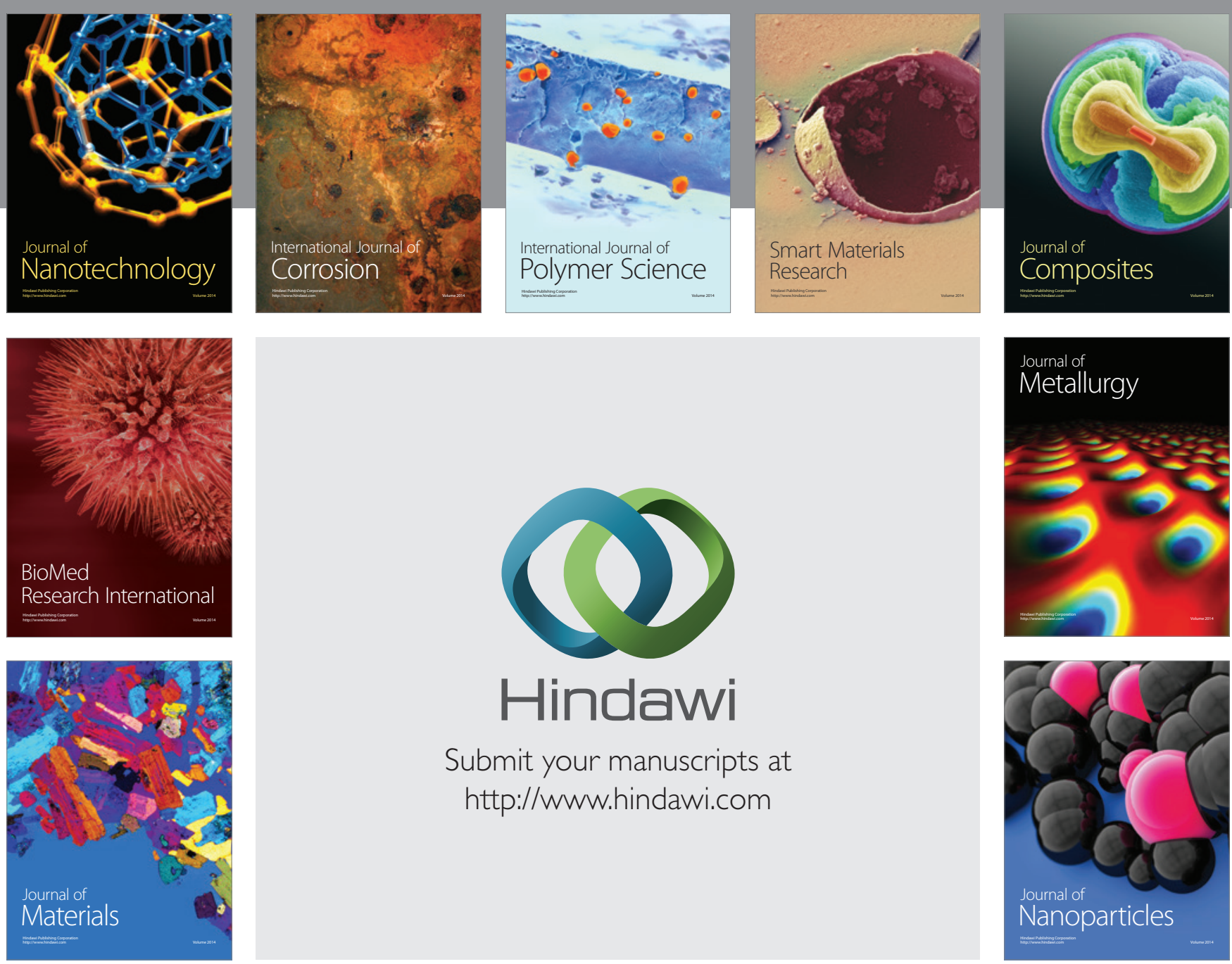

Submit your manuscripts at http://www.hindawi.com
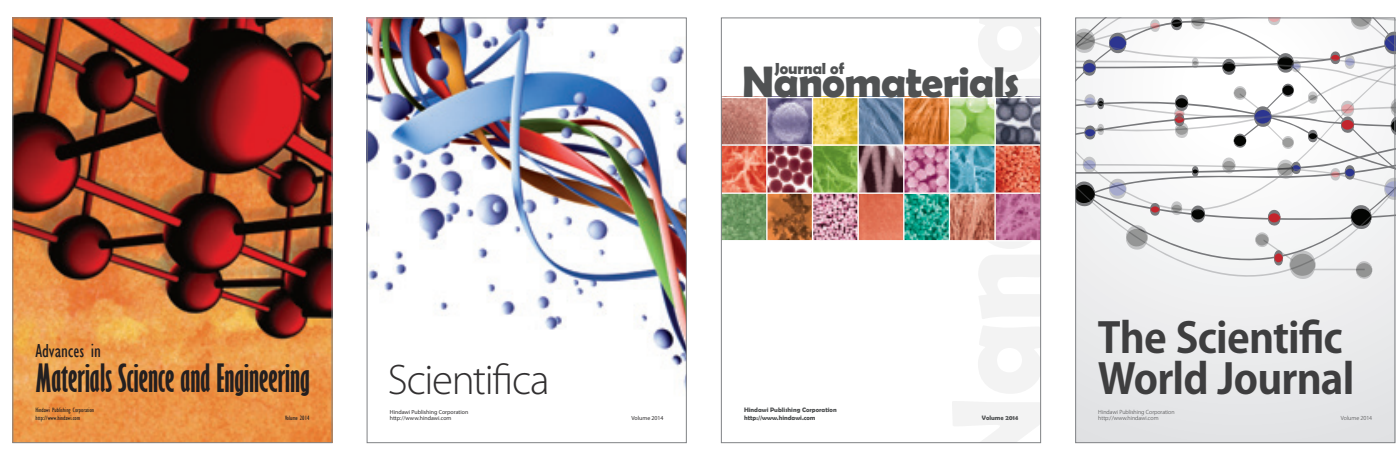

\section{The Scientific World Journal}
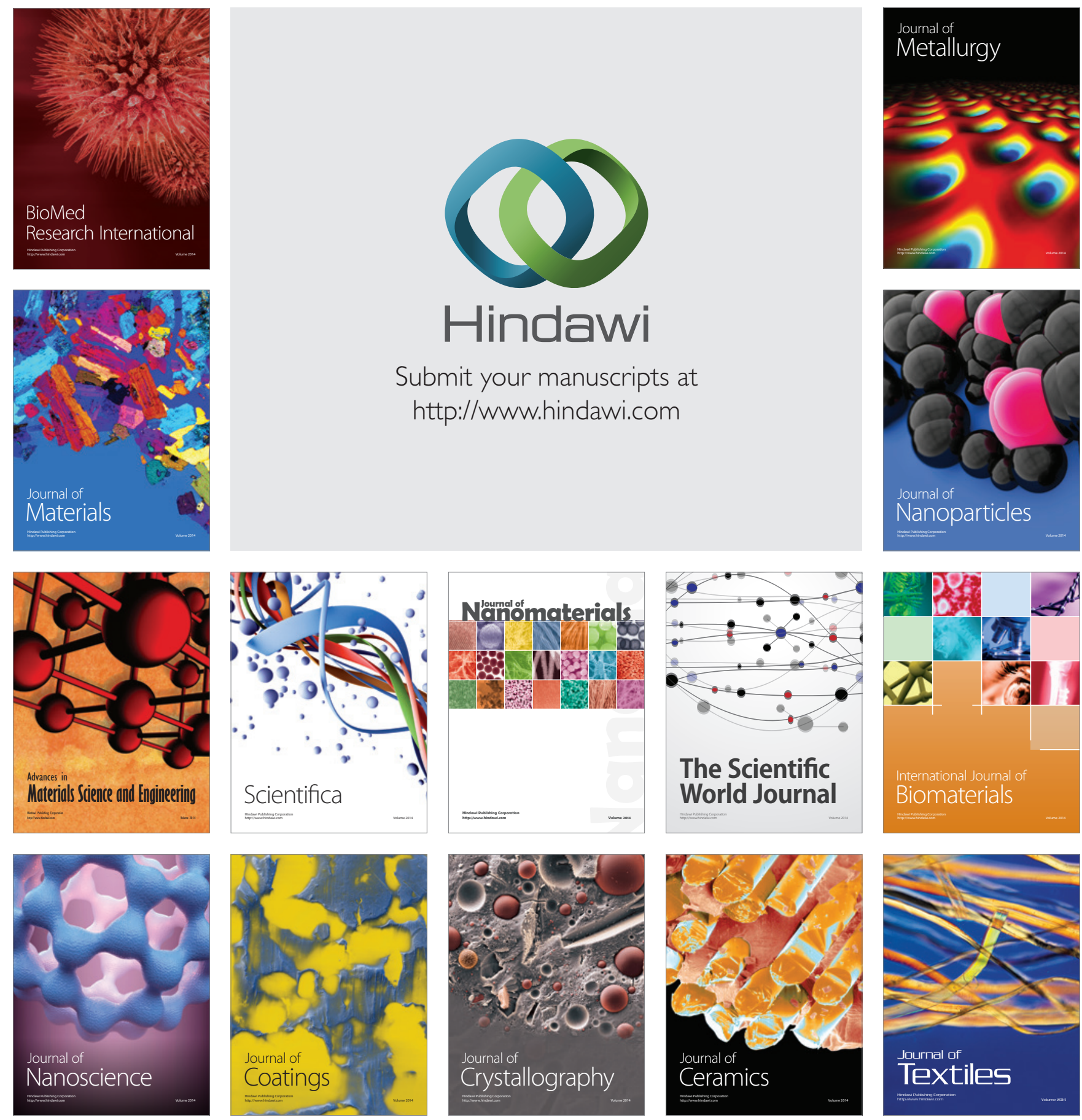